\title{
ICU Mortality is Increased in Septic Shock Patients Accompanied with Hypo- or Hyper- Serum Osmolarity: A Retrospective Study
}

\section{Gang Heng}

PLA Middle Military Command General Hospital https://orcid.org/0000-0001-6916-7480

Jiasi Zhang

Southwest Hospital

Benqi Huang

Hongkong univerisity of science and technology

Yanbing Shen

PLA Middle Military Command General Hospital

\section{Zhonghu Li}

PLA Middle Military Command General Hospital

Jiankun Jia

PLA Middle Military Command General Hospital

Chengcheng Zhang

Southwest Hospital

Jianxin Zhang

PLA Middle Military Command General Hospital

Tao Fu

PLA Middle Military Command General Hospital

Weidong Jin ( $\sim$ jwd202110@163.com )

PLA Middle Military Command General Hospital https://orcid.org/0000-0002-2939-6278

\section{Research}

Keywords: Serum osmolarity, Septic shock, ICU mortality

Posted Date: November 1st, 2021

DOI: https://doi.org/10.21203/rs.3.rs-1024500/v1

License: (1) (1) This work is licensed under a Creative Commons Attribution 4.0 International License.

Read Full License 


\section{ICU mortality is increased in septic shock patients accompanied with hypo- or hyper- serum osmolarity: A retrospective study}

Gang Heng ${ }^{* 1}$, Jiasi Zhang*2 ${ }^{*}$ Benqi Huang ${ }^{3}$, Yanbing Shen ${ }^{1}$, Zhonghu Li $^{1}$, Jiankun Jia ${ }^{1}$, Chengcheng Zhang ${ }^{4}$, Jianxin Zhang ${ }^{\# 1}$, Tao $\mathrm{Fu}^{\# 1}$ and Weidong Jin ${ }^{\# 1}$

* These authors contributed equally to this work

Correspondence to:

Prof. Weidong Jin, Department of general surgery, General hospital of central theater command, Wuhan 430000, China. Tel: 86-27-50772643; E-mail: jwd202110@163.com Or Prof. Tao Fu, E-mail: surgfu@ sina.com, or Prof. Jianxin Zhang, E-mail: mai_andy0@126.com

Statements and Declarations: The authors declare no potential conflicts of interest.

Take-home message: Serum osmolarity is an easy and reliable factor to predict ICU mortality of septic shock patients. Correction of hyper-osmolarity could lead to a reduction in the mortality. 


\begin{abstract}
Purpose: While many factors that are associated with increased mortality in septic shock patients have been identified, the effect of serum osmolarity to altering patient outcomes among ICU patients with septic shock has not been studied. This study was designed to examine the association of serum osmolarity with ICU 28-day mortality in that population.
\end{abstract}

Methods: The MIMIC-IV database was employed to identify patients diagnosed with septic shock. The serum osmolarity was calculated according to the serum concentration of $\mathrm{Na}^{+}, \mathrm{K}^{+}$, glucose and urea nitrogen synchronously. The statistical approaches used included multivariate logistic regression, propensity score analysis, inverse probability-weighting and causal mediation analysis.

Results: In this study, significant difference of 28-day mortality was observed in septic shock patients accompanied with hypo-osmolarity, hyper-osmolarity and normal osmolarity $(30.8 \%, 35.0 \%$ and $23.0 \%, \mathrm{P}<0.001)$ which was detected at ICU admission. We also found that transforming the hyper-osmolarity into normal osmolarity by fluid therapy in day 2 and day 3 would decrease this mortality.

Conclusion: Serum abnormality is significantly associated with increased 28-day mortality in septic shock patients.

Key words: Serum osmolarity, Septic shock, ICU mortality 


\section{Introduction}

Sepsis is a complex disorder in intensive care unit (ICU) and has severe health and economic burden on the patient and healthcare systems worldwide [1, 2]. According to the latest definition (Sepsis-3), septic shock is defined as a subset of sepsis in which underlying circulatory, cellular and metabolic abnormalities are profound enough to substantially increase the risk of mortality $[2,3]$. As concluded by Jean-Louis V et.al, the frequency of septic shock was estimated at $10.4 \%$ in studies reporting values for patients diagnosed at ICU admission and the mean ICU mortality of septic shock patients was $37.3 \%$ [3].

Over the past decades, a substantial amount of risk factors have been identified to predict the ICU mortality of septic shock patients, including serum albumin level, central venous pressure measurement, sequential organ failure assessment (SOFA) score, acute physiology and chronic health evaluation II(APACHE II) score and simplified acute physiology score II (SAPS II) [4-8]. However, the role of admission serum osmolarity which reflects the distribution of extracellular and intracellular water distribution, has not been studied in septic shock patients.

Serum osmolarity mainly depends on the concentration of $\mathrm{Na}^{+}, \mathrm{K}^{+}$, glucose and urea nitrogen, and is strongly associated with various body fluid balance [9]. The relativity of serum osmolarity and disease severity or hospital mortality has already been studied in several patient populations like those with stroke, intracranial hemorrhage, acute coronary syndrome, pulmonary disease and those admitted in the ICU [10-15]. Despite 
the consistency of clinical results indicating that serum osmolarity abnormalities are associated with increased hospital mortality, these conclusions are still not applicable to septic shock patients.

In this study, we conducted a retrospective study investigating the relationship between admission serum osmolarity and ICU mortality in patients with septic shock, collected in the MIMIC-IV database. Moreover, we studied whether the correction of serum osmolarity abnormality after admission could have a positive effect on the outcome of these patients.

\section{Methods}

\section{Study population}

This study is a retrospective study collected data from the Medical Information Mart for Intensive Care IV (MIMIC-IV version 0.4) database [16]. The author Gang H acquired the access of this database (Certification number 39516115). Firstly, 13604 patients diagnosed with sepsis were collected from this database. Then, we excluded patients who were not admitted into the ICU for the first time and those younger than 18 years old. Also, we excluded sepsis patients without septic shock and 5055 patients were preliminarily included in this study. Afterwards, according to the latest sepsis-3 criteria, 69 patients with sofa score lower than 2 points, and 279 patients with ICU stay shorter than 24 hours were excluded. Therefore, 4707 septic shock patients met the criteria and included in the final study. 
In our study, the patients' age, gender, weight, comorbidities, type of ICU admission, mean arterial pressure (MAP), heart rate, temperature, respiratory rate, oxygen saturation (SpO2), white blood cell (WBC), hemoglobin, platelet, sodium $\left(\mathrm{Na}^{+}\right)$, potassium $\left(\mathrm{K}^{+}\right)$, glucose, urea nitrogen, creatinine, albumin and lactate detected at the ICU admission were included.

\section{Calculation of serum osmolarity}

Serum osmolarity was calculated using the equation $\left(\mathrm{Na}^{+}+\mathrm{K}^{+}\right) \times 2+($ glucose $/ 18)+$ (urea/2.8). Only values of each element measured at the same time were used in this study. In this study, 290-309 $\mathrm{mmol} / \mathrm{L}$ was used as the normal range and reference group. Hyperosmolarity (> $309 \mathrm{mmol} / \mathrm{L})$ was categorized into two groups $(310-319 \mathrm{mmol} / \mathrm{L}$ and $>319 \mathrm{mmol} / \mathrm{L}$ ) in order to further examine the effect of hyperosmolarity in septic shock patients.

\section{Primary and secondary outcomes}

The primary outcome of this study is the ICU 28-day mortality. The secondary outcomes included the hospital mortality, length of ICU stay, volume of fluids administration, volume of crystalloids administration, volume of urine output, colloids intake, blood products intake, albumin intake, vasopressors-free days, ventilation-free days and AKI incidence. 


\section{Statistical analysis}

Continuous variables are presented as mean and standard error (SE) or median and interquartile range (IQR). Categorical variables are presented as numbers and percentages. Comparisons between groups are made using Student's t test or analysis of variance or Kruskal-Wallis test for continuous variables and $\mathrm{X}^{2}$ test for categorical variables.

Multivariate regression was selected to characterize the relationship between serum osmolarity and ICU mortality. Baseline characteristics such as age, gender, weight, sofa score, MAP, heart rate, hypertension, respiratory rate, temperature, $\mathrm{SpO} 2$, wbc, plt, hemoglobin, bun, creatinine, lactate, serum osmolarity group, use of albumin, use of colloids, use of blood products were selected to enter the multivariate logistic regression model (stepwise backward elimination method). In this study, the loss of variables was replaced by the mean value if the proportion of lost values was less than $10 \%$, or replaced by values within adjacent 2 days firstly if this proportion ranged from $10 \%$ to $20 \%$. The value would be abandoned if the percentage of loss was larger than $20 \%$.

Except for multivariate logistic regression, propensity score matching (PSM) and propensity score based inverse probability of treatment weighing (IPTW) were used to adjust the covariates to ensure the robustness of our finding. 1:1 nearest neighbor matching with a caliper width of 0.05 was applied, and the IPTW model was created using the estimated propensity scores as weights. Afterwards, the logistic regression 
was performed on the matched and weighted cohort separately, while the outcome was generated from the matched cohort.

Causal mediation analysis (CMA) produces an average causal mediation effect (ACME), average direct effect (ADE) and total effect on the outcome mediating through a mediator. In this study, we explored whether the effect of serum osmolarity on ICU mortality was proportionally mediated by the $\mathrm{SpO} 2$ and serum lactate.

\section{Results}

\section{Baseline characteristics}

In this study, a total of 4707 patients diagnosed with septic shock were enrolled, and the flow diagram of cohort selection was shown in Fig. 1. The baseline characteristics of survivors and non-survivors were presented in Table 1. The non-survivors had significantly higher sofa score (11.5 vs 8.5$)$ and lactate (5.5 vs 3.7$)$. Also, the serum osmolarity was statistically higher (307.3 vs 304.7 ) in non-survivor group, compared with that in survivor group.

\section{Primary and secondary outcomes}

Serum osmolarity was categorized into 4 groups and outcomes were compared within these groups using chi-square test or Kruskal-Wallis test. As shown in table 2, the ICU 28-day mortality was significantly lower in normal serum osmolarity group (290-309 
mmol/L) than hypo-osmolartity $(<290 \mathrm{mmol} / \mathrm{L})$ and hyper-osmolarity (310-319 $\mathrm{mmol} / \mathrm{L}$ and $>319 \mathrm{mmol} / \mathrm{L})(23.0 \%$ vs $30.8 \%, 33.9 \%$ and $36.0 \%, \mathrm{P}<0.001)$. This difference was still observed in hospital mortality of septic shock patients. However, the difference of ICU length of stay (LOS) was not significant in 4 groups $(\mathrm{P}=0.249)$.

In this study, we also compared albumin intake, fluid intake (crystalloids intake, colloids intake and colloids intake), urine output, vasopressor-free days, ventilationfree days and acute kidney injure (AKI) incidence in these groups and the detailed results were presented in the Table 2. Compared with hyper-osmolarity group, less fluid was administrated in hypo and normal osmolarity patients (day 1 and day $2, \mathrm{P}<0.01$ ), while more urine was produced (day 1,2 and $3, \mathrm{P}<0.01$ ). Besides, the proportion of patients who achieved albumin, colloids and blood products infusion was statistically higher in hypo-osmolarity group $(\mathrm{P}<0.01)$, while the AKI incidence within first 24 hours was significantly higher in hyper-osmolarity group.

\section{Sensitivity analysis}

The multivariate logistic regression was used to identify the relationship between serum osmolarity and ICU mortality, and the result showed that both hypo-osmolarity and hyper-osmolarity were significantly associated with ICU mortality (OR 1.48, p=0.002 and OR $1.28 \mathrm{p}=0.009)$. Except for multivariate logistic regression, the propensity score matching model (PSM) and inverse probability of treatment weighing (IPTW) were also used to verify this relationship, and the same trend of association was observed 
(Table 3).

Causal mediation analysis (CMA) showed that the SpO2 mediated $4.90 \%$ (95\% CI $1.99 \%-7.85 \% ; \mathrm{P}=0.002$ ) of the effect of serum osmolarity in ICU mortality, and the level of lactate at admission mediated $5.41 \%(95 \%$ CI $2.40 \%-8.22 \% ; \mathrm{P}=0.002)$ of this effect (Fig. 2).

\section{Correction of abnormal serum osmolarity decrease the ICU mortality}

In this study, we also presented the dynamics of mortality and serum osmolarity within 3 days after ICU admission (Fig. 3). As shown in figure 3A, those patients with hypoosmolarity at admission transformed to normal osmolarity in day 2 and day 3 after treatment did not show significant decrease of mortality, compared with those remained in hypo-osmolarity. However, this trend did not exist in normal and hyper-osmolarity group. In normal osmolarity group, the ICU mortality was statistically lower in patients maintaining normal osmolarity or transforming to hypo-osmolarity, compared with those transformed to hyper-osmolarity in day 2 and day $3(\mathrm{P}<0.001)$ (Fig. 3B). Obviously, in the hyper-osmolarity group, patients who achieved the normal osmolarity in day 2 and day 3 had a statistically lower ICU mortality $(\mathrm{P}<0.001$ and $\mathrm{P}<0.01)$ than those patients remaining hyper-osmolarity (Fig. 3C). 


\section{Discussion}

Although many previous studies have studied the association between serum osmolarity and mortality in critically ill patients, emergency medical patients and patients with stroke, intracranial hemorrhage and acute coronary syndrome, there was limited study identifying the relationship between serum osmolarity and ICU mortality of septic shock patients. In this study, we demonstrated that abnormal serum osmolarity was associated with significantly higher ICU 28-day mortality than normal serum osmolarity for the first time, as well as higher hospital mortality. We also verified that correction of serum osmolarity into the normal range would decrease this mortality.

Fluid balance of the body is of vital importance for septic shock patients, and serum osmolarity plays a significant role in the distribution of intracellular and extracellular fluid distribution $[11,17]$. As reported, perturbation of serum osmolarity is common in patients who are admitted into the ICU, which leads to the disturbance of body's internal environment, potentially resulting in adverse outcomes [18]. Hyper-osmolarity could lead to the mobilization of fluid from venous capacitance vessels to the circulatory volume, thereby aggravating the hypoxia of organs or tissues [19, 20]. Besides, hyperosmolarity is always accompanied by hypernatraemia or hyperglycaemia, which have been reported as separate risk factors for cardiac mortality [21, 22]. In previous study, hypo-osmolarity on admission were also significantly associated with increased mortality in critically ill and emergency patients [18]. However, the exact pathophysiological mechanism of hypo-osmolarity on increased mortality was still unknown. 
For septic shock patients, hemodynamic instability is widely recognized as a risk factor for mortality, and many approaches have been practiced to detect and then reverse that instability $[23,24]$. Blood pressure, mean arterial pressure and lactate concentration are three major factors indicating the fluid balance or tissue perfusion of the body, and plenty of studies have focused on them [25-27]. However, except for these factors, serum osmolarity is also an effective one to identify the instability of fluid balance. The serum osmolarity could simply reflect substantial organ dysfunction or derangement of overall homeostatic mechanisms for salt, glucose and urea in particular, thereby it is a very easy score to calculate, based on the data that are generally available for most hospital admissions. Besides, we have identified the treatment or correction of the hyper-osmolarity detected on admission could lead to a reduction in mortality among septic shock patients, indicating that serum osmolarity could serve as a credible method to predict ICU mortality of these patients.

Although this study is the first one to investigate the relationship between serum osmolarity and ICU mortality of patients diagnosed as septic shock, and it is also firstly study the effect that correction of serum osmolarity abnormality have on ICU mortality, it still have several limitations. First, this is a retrospective study which could not strictly balance the baseline characteristics of patients with different serum osmolarity categories. Second, the serum osmolarity in the present study was calculated rather than being measured directly, which could result in deviation from actual serum osmolarity values despite the optimal calculation equation and simultaneous values of components were considered. Therefore, to further explore the relationship between serum 
osmolarity and ICU mortality of septic shock patients, a larger and prospective study should be performed.

\section{Conclusion}

In conclusion, through the analysis of a large clinical database, our study indicates that both hypo-osmolarity and hyper-osmolarity at ICU admission were associated with increased mortality in patients with septic shock. Moreover, correction of hyperosmolarity into the normal level would decreased this mortality.

\section{Acknowledgements}

None

\section{Author details}

${ }^{1}$ Department of general surgery, General hospital of central theater command, Wuhan 430000, China; ${ }^{2}$ Center of Haematology, Southwest Hospital, Third Military Medical University (Army Medical University), Chongqing 400038, China; ${ }^{3}$ Department of science, Hong Kong university of Science and Technology, Clear Water Bay, Kowloon, Hong Kong, China; ${ }^{4}$ Department of Hepatobiliary Surgery, Southwest Hospital, Third Military Medical University (Army Medical University), Chongqing 400038, China; 


\section{Author contribution}

Jianxin Zhang, Tao Fu and Weidong Jin designed the study. Gang Heng and Jiasi Zhang performed the research and wrote the article. Benqi Huang, Yanbing Shen, Zhonghu Li, Jiankun Jia and Chengcheng Zhang analysed the data and conducted primary statistical analysis.

\section{Competing interests}

The authors declare that they have no competing interests.

\section{Funding}

None

\section{Availability of supporting data}

The datasets presented in the current study are available in the MIMIC-IV database (https://physionet.org/content/mimiciv/0.4/)

\section{Ethics approval and consent to participate}

The establishment of this database was approved by the Massachusetts Institute of

Technology (Cambridge, MA) and Beth Israel Deaconess Medical Center (Boston, 
MA), and consent was obtained for the original data collection. Therefore, the ethical approval statement and the need for informed consent were waived for this manuscript.

\section{Consent for publication}

Not applicable

\section{Reference:}

1. Cecconi M, Evans L, Levy M, Rhodes A, (2018) Sepsis and septic shock. The Lancet 392: 75-87

2. Rhodes A, Evans LE, Alhazzani W, Levy MM, Antonelli M, Ferrer R, Kumar A, Sevransky JE, Sprung CL, Nunnally ME, Rochwerg B, Rubenfeld GD, Angus DC, Annane D, Beale RJ, Bellinghan GJ, Bernard GR, Chiche JD, Coopersmith C, De Backer DP, French CJ, Fujishima S, Gerlach H, Hidalgo JL, Hollenberg SM, Jones AE, Karnad DR, Kleinpell RM, Koh Y, Lisboa TC, Machado FR, Marini JJ, Marshall JC, Mazuski JE, McIntyre LA, McLean AS, Mehta S, Moreno RP, Myburgh J, Navalesi P, Nishida O, Osborn TM, Perner A, Plunkett CM, Ranieri M, Schorr CA, Seckel MA, Seymour CW, Shieh L, Shukri KA, Simpson SQ, Singer M, Thompson BT, Townsend SR, Van der Poll T, Vincent JL, Wiersinga WJ, Zimmerman JL, Dellinger RP, (2017) Surviving Sepsis Campaign: International Guidelines for Management of Sepsis and Septic Shock: 2016. Intensive care medicine 43: 304-377

3. Vincent JL, Jones G, David S, Olariu E, Cadwell KK, (2019) Frequency and mortality of septic shock in Europe and North America: a systematic review and meta-analysis. Critical care 23: 196

4. Zhou S, Zeng Z, Wei H, Sha T, An S, (2021) Early combination of albumin with crystalloids administration might be beneficial for the survival of septic patients: a retrospective analysis from MIMIC-IV database. Annals of intensive care 11: 42

5. Chen H, Zhu Z, Zhao C, Guo Y, Chen D, Wei Y, Jin J, (2020) Central venous pressure measurement is associated with improved outcomes in septic patients: an analysis of the MIMIC-III database. Critical care 24: 433

6. Arabi Y, Al Shirawi N, Memish Z, Venkatesh S, Al-Shimemeri A, (2003) Assessment of six mortality prediction models in patients admitted with severe sepsis and septic shock to the intensive care unit: a prospective cohort study. Critical care 7: R116-122

7. Huang CT, Ruan SY, Tsai YJ, Ku SC, Yu CJ, (2019) Clinical Trajectories and Causes of Death in Septic Patients with a Low APACHE II Score. Journal of clinical medicine 8

8. Pereira B, Dorigatti A, Melek M, Dos Santos J, Ferreira M, Calderan T, Carmona C, Fraga G, (2019) Septic shock patients admitted to the intensive care unit with higher SOFA score tend to have 
higher incidence of abdominal compartment syndrome - a preliminary analysis. Anaesthesiology intensive therapy 51: 370-372

9. Gennari FJ, (1984) Current concepts. Serum osmolality. Uses and limitations. The New England journal of medicine 310: 102-105

10. Yagi T, Kaneko T, Tsuruta R, Kasaoka S, Miyauchi T, Fujita M, Kawamura Y, Sakka SG, Maekawa T, (2011) Global end-diastolic volume, serum osmolarity, and albumin are risk factors for increased extravascular lung water. Journal of critical care 26: 224 e229-213

11. Nicholson T, Bennett K, Silke B, (2012) Serum osmolarity as an outcome predictor in hospital emergency medical admissions. European journal of internal medicine 23: e39-43

12. Holtfreter B, Bandt C, Kuhn SO, Grunwald U, Lehmann C, Schutt C, Grundling M, (2006) Serum osmolality and outcome in intensive care unit patients. Acta anaesthesiologica Scandinavica 50: $970-977$

13. Nag C, Das K, Ghosh M, Khandakar MR, (2012) Plasma osmolality in acute spontanious intracerebral hemorrhage: Does it influence hematoma volume and clinical outcome? Journal of research in medical sciences : the official journal of Isfahan University of Medical Sciences 17: 548-551

14. Rohla M, Freynhofer MK, Tentzeris I, Farhan S, Wojta J, Huber K, Weiss TW, (2014) Plasma osmolality predicts clinical outcome in patients with acute coronary syndrome undergoing percutaneous coronary intervention. European heart journal Acute cardiovascular care 3: 8492

15. Bhalla A, Sankaralingam S, Dundas R, Swaminathan R, Wolfe CD, Rudd AG, (2000) Influence of raised plasma osmolality on clinical outcome after acute stroke. Stroke 31: 2043-2048

16. Johnson A, Bulgarelli L, Pollard T, Horng S, Celi L A, Mark R. MIMIC-IV (version 0.4). PhysioNet. 2020. Available from: https://doi.org/10.13026/a3wn-hq05.

17. El-Sharkawy AM, Watson P, Neal KR, Ljungqvist O, Maughan RJ, Sahota O, Lobo DN, (2015) Hydration and outcome in older patients admitted to hospital (The HOOP prospective cohort study). Age and ageing 44: 943-947

18. Shen Y, Cheng X, Ying M, Chang HT, Zhang W, (2017) Association between serum osmolarity and mortality in patients who are critically ill: a retrospective cohort study. BMJ open 7: e015729

19. El-Sharkawy AM, Devonald MAJ, Humes DJ, Sahota O, Lobo DN, (2020) Hyperosmolar dehydration: A predictor of kidney injury and outcome in hospitalised older adults. Clinical nutrition 39: 2593-2599

20. Arevalo-Lorido JC, Gomez JC, Formiga F, Conde-Martel A, Carrera-Izquierdo M, Muela-Molinero A, Davila-Ramos MF, Serrado-Iglesias A, Manzano-Espinosa L, Montero-Perez-Barquero M, (2016) High serum osmolarity at admission determines a worse outcome in patients with heart failure: Is a new target emerging? International journal of cardiology 221: 238-242

21. Xie J, Cui K, Hao H, Zhang Y, Lin H, Chen Z, Huang X, Cao S, Liao W, Bin J, Kitakaze M, Liao Y, (2016) Acute hyperglycemia suppresses left ventricular diastolic function and inhibits autophagic flux in mice under prohypertrophic stimulation. Cardiovascular diabetology 15: 136

22. Wannamethee SG, Shaper AG, Lennon L, Papacosta O, Whincup P, (2016) Mild hyponatremia, hypernatremia and incident cardiovascular disease and mortality in older men: A populationbased cohort study. Nutrition, metabolism, and cardiovascular diseases : NMCD 26: 12-19

23. Cecconi M, De Backer D, Antonelli M, Beale R, Bakker J, Hofer C, Jaeschke R, Mebazaa A, Pinsky 
MR, Teboul JL, Vincent JL, Rhodes A, (2014) Consensus on circulatory shock and hemodynamic monitoring. Task force of the European Society of Intensive Care Medicine. Intensive care medicine 40: 1795-1815

24. Holmes CL, Walley KR, Chittock DR, Lehman T, Russell JA, (2001) The effects of vasopressin on hemodynamics and renal function in severe septic shock: a case series. Intensive care medicine 27: 1416-1421

25. Asfar P, Meziani F, Hamel JF, Grelon F, Megarbane B, Anguel N, Mira JP, Dequin PF, Gergaud S, Weiss N, Legay F, Le Tulzo Y, Conrad M, Robert R, Gonzalez F, Guitton C, Tamion F, Tonnelier JM, Guezennec P, Van Der Linden T, Vieillard-Baron A, Mariotte E, Pradel G, Lesieur O, Ricard JD, Herve F, du Cheyron D, Guerin C, Mercat A, Teboul JL, Radermacher P, Investigators S, (2014) High versus low blood-pressure target in patients with septic shock. The New England journal of medicine 370: 1583-1593

26. Bourgoin A, Leone M, Delmas A, Garnier F, Albanese J, Martin C, (2005) Increasing mean arterial pressure in patients with septic shock: effects on oxygen variables and renal function. Critical care medicine 33: 780-786

27. Casserly B, Phillips GS, Schorr C, Dellinger RP, Townsend SR, Osborn TM, Reinhart K, Selvakumar $\mathrm{N}$, Levy MM, (2015) Lactate measurements in sepsis-induced tissue hypoperfusion: results from the Surviving Sepsis Campaign database. Critical care medicine 43: 567-573 
Table 1: Comparison of baseline characteristics between survivors and non-survivors

\begin{tabular}{|c|c|c|c|c|}
\hline Variables & $\operatorname{Total}(\mathrm{n}=4707)$ & Survivors ( $\mathrm{n}=3392$ ) & Non-survivors $(\mathrm{n}=1315)$ & $P$ value \\
\hline Age (years) & $67.8 \pm 0.2$ & $66.7 \pm 0.3$ & $70.6 \pm 0.4$ & $<0.001$ \\
\hline Male (n (\%)) & $2602(55.3 \%)$ & $1894(55.8 \%)$ & $708(53.8 \%)$ & 0.259 \\
\hline Weight (kg) & $83.6 \pm 0.4$ & $83.9 \pm 0.4$ & $82.8 \pm 0.9$ & 0.338 \\
\hline SOFA score & $9.4 \pm 0.1$ & $8.5 \pm 0.1$ & $11.5 \pm 0.1$ & $<0.001$ \\
\hline \multicolumn{5}{|l|}{ Comorbidities } \\
\hline COPD (n (\%)) & $495(10.5 \%)$ & $371(10.9 \%)$ & $124(9.4 \%)$ & 0.130 \\
\hline $\mathrm{CAD}(\mathrm{n}(\%))$ & $1041(22.1 \%)$ & $769(22.7 \%)$ & $272(20.7 \%)$ & 0.141 \\
\hline Hypertension (n (\%)) & $1705(36.2 \%)$ & $1283(37.8 \%)$ & $422(32.1 \%)$ & $<0.001$ \\
\hline Type of ICU & & & & $<0.001$ \\
\hline Medical (n (\%)) & $2787(59.2 \%)$ & $2009(59.2 \%)$ & $778(59.2 \%)$ & \\
\hline Surgical (n (\%)) & $519(11.0 \%)$ & $388(11.4 \%)$ & $131(10.0 \%)$ & \\
\hline Coronary (n (\%)) & $512(10.9 \%)$ & $332(9.8 \%)$ & $180(13.7 \%)$ & \\
\hline Cardiac (n (\%)) & $338(7.2 \%)$ & $268(7.9 \%)$ & $70(5.3 \%)$ & \\
\hline Trauma (n (\%)) & $473(10.0 \%)$ & $341(10.1 \%)$ & $132(10.0 \%)$ & \\
\hline \multicolumn{5}{|l|}{ Vital signs } \\
\hline $\mathrm{MAP}(\mathrm{mmHg})$ & $51.6 \pm 0.2$ & $52.6 \pm 0.2$ & $48.8 \pm 0.4$ & $<0.001$ \\
\hline Heart rate & $90.5 \pm 0.3$ & $89.5 \pm 0.3$ & $93.4 \pm 0.5$ & $<0.001$ \\
\hline Respiratory rate & $21.1 \pm 0.1$ & $20.8 \pm 0.1$ & $22.0 \pm 0.1$ & $<0.001$ \\
\hline Temperature $\left({ }^{\circ} \mathrm{C}\right)$ & $36.9 \pm 0.0$ & $37.0 \pm 0.0$ & $36.7 \pm 0.0$ & $<0.001$ \\
\hline $\mathrm{SpO} 2$ & $96.6 \pm 0.0$ & $96.9 \pm 0.0$ & $96.0 \pm 0.1$ & $<0.001$ \\
\hline \multicolumn{5}{|l|}{ Laboratory tests } \\
\hline WBC & $15.3 \pm 0.2$ & $14.8 \pm 0.2$ & $16.5 \pm 0.4$ & $<0.001$ \\
\hline Hemoglobin & $10.4 \pm 0.0$ & $10.5 \pm 0.0$ & $10.2 \pm 0.1$ & $<0.001$ \\
\hline Platelet & $198.6 \pm 1.7$ & $201.7 \pm 2.0$ & $190.7 \pm 3.3$ & 0.005 \\
\hline BUN & $36.7 \pm 0.4$ & $34.7 \pm 0.5$ & $42.1 \pm 0.8$ & $<0.001$ \\
\hline Creatinine & $1.9 \pm 0.0$ & $1.8 \pm 0.0$ & $2.2 \pm 0.0$ & $<0.001$ \\
\hline Glucose & $162.1 \pm 1.5$ & $162.0 \pm 1.8$ & $162.7 \pm 2.8$ & 0.822 \\
\hline Lactate & $4.2 \pm 0.5$ & $3.7 \pm 0.0$ & $5.5 \pm 0.1$ & $<0.001$ \\
\hline Albumin & $3.0 \pm 0.0$ & $3.1 \pm 0.0$ & $2.8 \pm 0.0$ & $<0.001$ \\
\hline Osmolarity & $305.4 \pm 0.3$ & $304.7 \pm 0.3$ & $307.3 \pm 0.6$ & $<0.001$ \\
\hline
\end{tabular}


Table 2: Characteristics and outcomes by serum osmolarity categories

\begin{tabular}{|c|c|c|c|c|c|}
\hline \multirow[b]{2}{*}{ Outcomes } & \multicolumn{5}{|c|}{ Serum osmolarity categories $(\mathrm{mmol} / \mathrm{L})$} \\
\hline & $<290(\mathrm{n}=633)$ & $290-309(n=2536)$ & $310-319(n=826)$ & $>319(\mathrm{n}=712)$ & $P$ value \\
\hline ICU mortality (n (\%)) & $195(30.8 \%)$ & $593(23.0 \%)$ & $282(33.9 \%)$ & $256(36.0 \%)$ & $<0.001$ \\
\hline Hospital mortality (n (\%)) & $205(32.4 \%)$ & $629(24.8 \%)$ & $292(35.4 \%)$ & $268(37.6 \%)$ & $<0.001$ \\
\hline ICU LOS (median (IQR)) & $9.3(4.9-17.9)$ & $9.9(5.7-18.0)$ & $10.1(5.4-18.5)$ & $9.8(4.8-17.3)$ & 0.249 \\
\hline Albumin intake within 3 days (n (\%)) & $213(33.6 \%)$ & $626(24.7 \%)$ & $170(20.6 \%)$ & $137(19.2 \%)$ & $<0.001$ \\
\hline Fluid intake in day $1(\mathrm{ml})$ & $9664.4 \pm 349.0$ & $10128.4 \pm 201.8$ & $10384.7 \pm 350.2$ & $13690.2 \pm 1128.8$ & $<0.001$ \\
\hline Fluid intake in day $2(\mathrm{ml})$ & $5375.3 \pm 228.3$ & $5113.4 \pm 98.5$ & $5443.7 \pm 170.3$ & $5741.8 \pm 191.4$ & 0.007 \\
\hline Fluid intake in day $3(\mathrm{ml})$ & $4789.8 \pm 214.1$ & $4448.9 \pm 95.2$ & $4866.8 \pm 188.3$ & $4519.2 \pm 177.1$ & 0.337 \\
\hline Urine output in day $1(\mathrm{ml})$ & $1766.5 \pm 58.6$ & $1758.7 \pm 34.4$ & $1450.8 \pm 51.2$ & $1404.8 \pm 62.3$ & $<0.001$ \\
\hline Urine output in day $2(\mathrm{ml})$ & $1553.8 \pm 62.4$ & $1603.5 \pm 39.7$ & $1440.3 \pm 68.4$ & $1360.8 \pm 55.9$ & $<0.001$ \\
\hline Urine output in day $3(\mathrm{ml})$ & $1653.1 \pm 67.2$ & $1761.0 \pm 38.3$ & $1637.0 \pm 69.1$ & $1550.0 \pm 64.2$ & 0.006 \\
\hline Crystalloids intake in day $1(\mathrm{ml})$ & $9077.0 \pm 328.1$ & $9639.4 \pm 187.4$ & $9892.0 \pm 331.5$ & $13116.9 \pm 1073.5$ & $<0.001$ \\
\hline Crystalloids intake in day $2(\mathrm{ml})$ & $5123.5 \pm 221.7$ & $4893.1 \pm 94.0$ & $5238.8 \pm 161.8$ & $5547.7 \pm 184.4$ & 0.003 \\
\hline Crystalloids intake in day $3(\mathrm{ml})$ & $4601.7 \pm 206.0$ & $4282.3 \pm 91.7$ & $4700.4 \pm 171.8$ & $4366.5 \pm 171.8$ & 0.295 \\
\hline Colloids intake within 3 days (n (\%)) & $354(55.9 \%)$ & $1216(47.9 \%)$ & $332(40.2 \%)$ & $276(38.8 \%)$ & $<0.001$ \\
\hline Blood products within 3 days (n $(\%)$ ) & $296(62.6 \%)$ & $1438(56.7 \%)$ & $445(53.9 \%)$ & $380(53.4 \%)$ & 0.002 \\
\hline Vasopressor-free in 28 days & $19.8 \pm 0.4$ & $20.5 \pm 0.2$ & $20.4 \pm 0.3$ & $21.0 \pm 0.3$ & 0.006 \\
\hline Ventilation-free in 28 days & $21.4 \pm 0.4$ & $22.2 \pm 0.2$ & $23.3 \pm 0.2$ & $23.6 \pm 0.2$ & 0.003 \\
\hline AKI within 24 hours (n (\%)) & $343(54.2 \%)$ & $1464(57.7 \%)$ & $542(65.6 \%)$ & $443(62.2 \%)$ & $<0.001$ \\
\hline
\end{tabular}

Table 3: Primary outcomes analysis with different models

\begin{tabular}{|c|c|c|c|c|}
\hline \multirow[b]{2}{*}{ Model } & \multirow{2}{*}{$\begin{array}{l}\text { Variables } \\
\text { Normal osmolarity } \\
(290-309)\end{array}$} & \multirow{2}{*}{$\begin{array}{l}\text { Odds ratio } \\
\text { Reference }\end{array}$} & \multirow[t]{2}{*}{$95 \% \mathrm{CI}$} & \multirow[t]{2}{*}{$\mathrm{P}$ value } \\
\hline & & & & \\
\hline \multirow[t]{2}{*}{ Multivariable logistic regression } & Hypo-osmolarity $(<290)$ & 1.71 & $1.38-2.13$ & $<0.001$ \\
\hline & Hyper-osmolarity (>309) & 1.28 & $1.09-1.50$ & 0.002 \\
\hline \multirow[t]{2}{*}{ IPTW } & Hypo-osmolarity $(<290)$ & 1.83 & $1.52-2.21$ & $<0.001$ \\
\hline & Hyper-osmolarity (>309) & 1.25 & $1.08-1.45$ & 0.005 \\
\hline \multirow[t]{2}{*}{ PSM } & Hypo-osmolarity $(<290)$ & 2.02 & $1.43-2.86$ & $<0.001$ \\
\hline & Hyper-osmolarity (>309) & 1.37 & $1.11-1.68$ & 0.003 \\
\hline
\end{tabular}




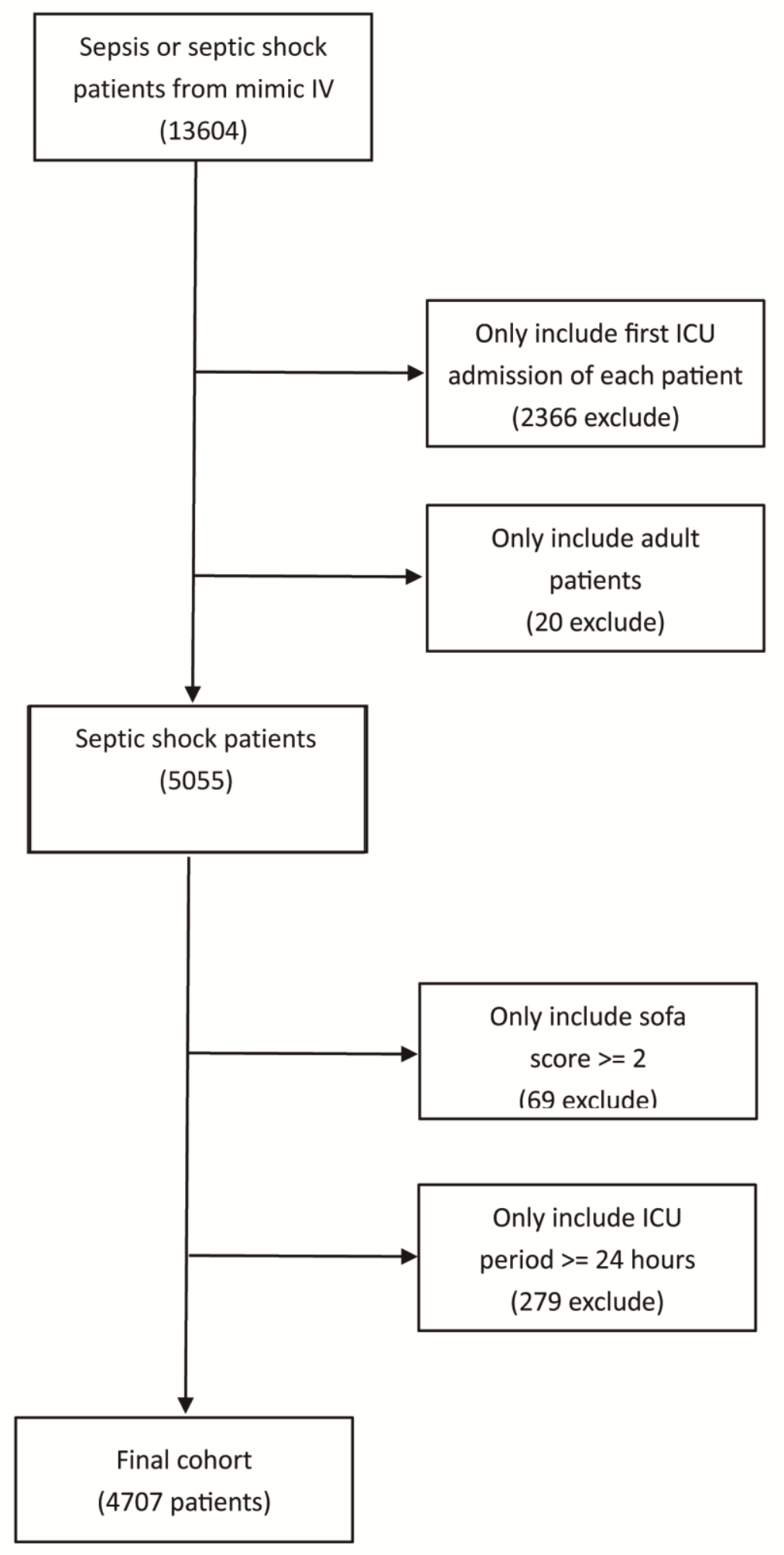

Fig. 1: Study flow diagram of the present study. 
B
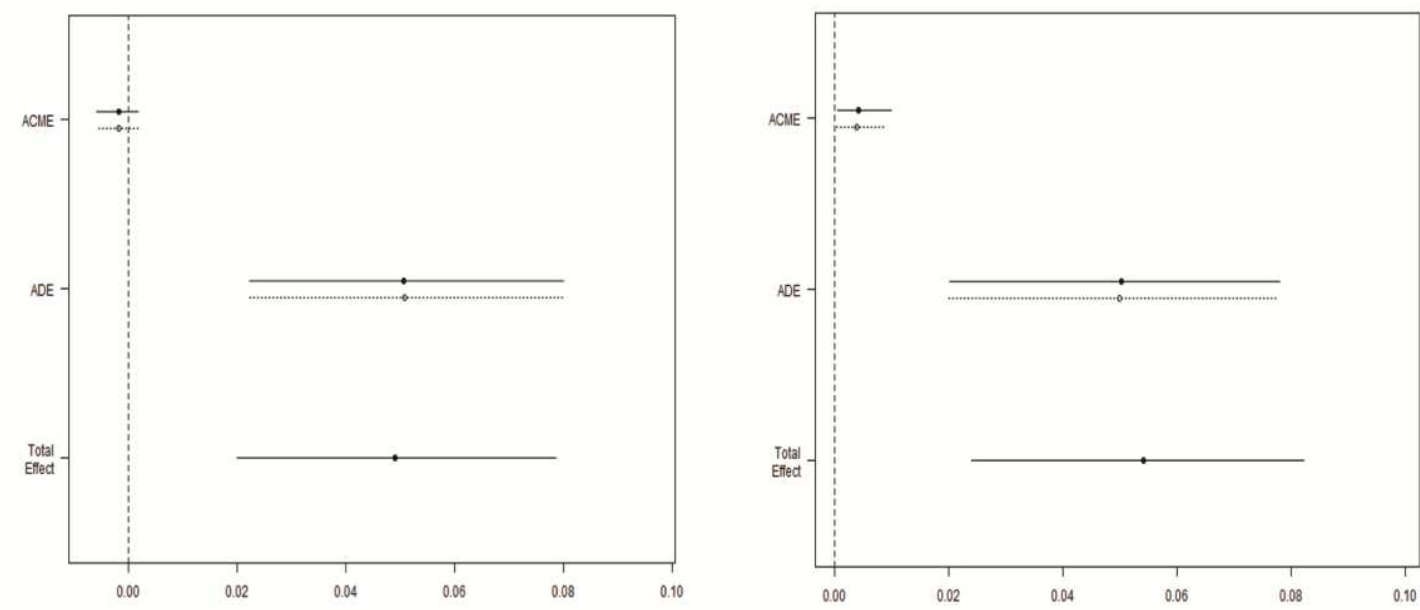

Fig. 2: Casual mediation analysis of $\mathrm{SpO} 2$ and lactate at admission. (A) The effect of $\mathrm{SpO} 2$ mediated of the serum osmolarity in ICU mortality. (B) The effect of lactate mediated of the serum osmolarity in ICU mortality. The solid line represents the normal osmolarity group, and the dashed line represents the abnormal osmolarity group. 
A

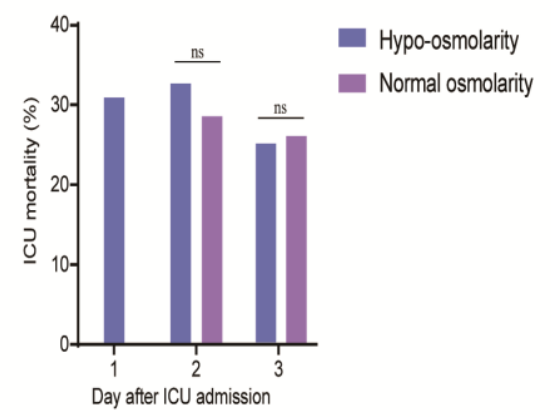

B

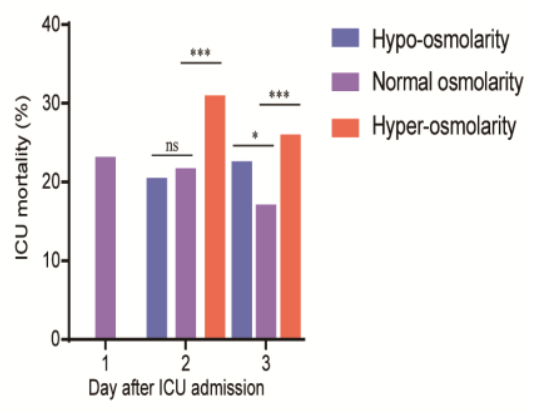

C

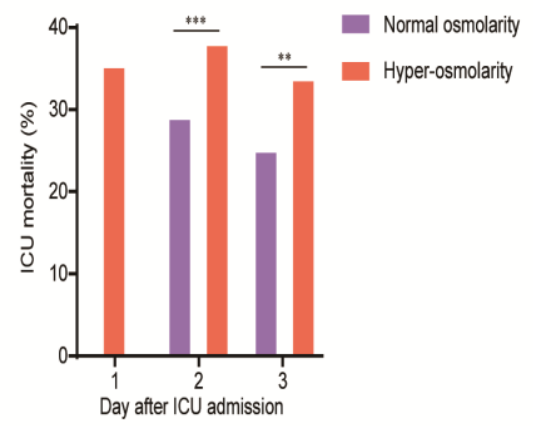

Fig. 3: The dynamics of mortality and serum osmolarity within 3 days after ICU admission. (A) The dynamical ICU mortality of these patients with hypo-osmolarity at admission transformed to normal osmolarity in day 2 and day 3. (B) The dynamical ICU mortality of these patients with normal osmolarity at admission transformed to hypo-, normal or hyper-osmolarity in day 2 and day 3. (C) The dynamical ICU mortality of these patients with hyper-osmolarity at admission transformed to normal osmolarity in day 2 and day 3. The mortality changes of patients who transformed to hyperosmolarity in hypo-osmolarity group and who transformed to hypo-osmolarity in hyper-osmolarity group were not presented as the number is in single digit, which could result in statistical bias. 